\title{
La organización política y social de los saharauis en España. Desarrollo y desafíos de las asociaciones de migrantes
}

Political and Social Organization of Saharawi People in Spain. Development and Challenges of Migrant Associations

\section{CARMEN GÓMEZ MARTÍN \\ FLACSO ECUADOR (ECUADOR) • gomez.martin.carmen@gmail.com}

Doctora en sociología por l'Ecole D’Hautes Études de Sciences Sociales de Paris, es profesora titular de FLACSO Ecuador. Ha publicado el libro La migración saharaui en España. Estrategias de visibilidad en el tercer tiempo del exilio.

RESUMEN: Este artículo profundiza en una temática poco conocida dentro de los estudios sobre el Sáhara Occidental: el proceso de organización social, política y cultural de los saharauis en el espacio migratorio. Más allá de la dificultad que ha tenido el desarrollo de las asociaciones de migrantes y de diversos colectivos de jóvenes saharauis en España desde principios de la década del 2000, y las tensiones que se han ido materializando después de su irrupción en el campo político saharaui, estas organizaciones son el reflejo de los profundos cambios que se han producido en la última década en el seno de la sociedad saharaui. Dichas transformaciones están relacionadas directamente con procesos de (re)cambio generacional y están obligando a las tradicionales estructuras del Frente Polisario a adaptarse a una nueva realidad.

PALABRAS CLAVE: Saharauis; España; migración; organización política, social, cultural; asociacionismo.
ABSTRACT: This article deals with a topic relatively unknown in studies on Western Sahara: the process of cultural social, political, and Sahrawis in the migration space. It analyzes the development of migrant associations and various groups of young Saharawis in Spain since the early $2000 \mathrm{~s}$, and the various tensions that have been materializing after its entry into the Saharawi political field. These organizations reflect the profound changes that have occurred in the last decade within Saharawi society. These transformations are directly related to processes of generational change and are forcing the traditional structures of the Polisario Front to adapt to a new reality.

KEYWORDS: Saharawi people; Spain; migration; political organization; associationism. 


\section{Introducción}

A pesar de que la migración desde los campamentos de refugiados saharauis hacia España se desencadenó hace ya más de veinte años, este proceso sigue siendo poco estudiado en el ámbito académico. La falta de atención hacia esta cuestión ha impedido que hasta el momento haya una comprensión mayor de la evolución que ha experimentado la sociedad saharaui y su dinamismo social interno. La problemática saharaui suele pensarse desde miradas estáticas o ancladas territorialmente a los campamentos de refugiados o a los territorios ocupados, desconectando e incluso aislando el propio devenir del Sáhara Occidental de procesos y dinámicas más globales, multiescalares y multiespaciales (Gómez 2016). Precisamente, la irrupción de nuevas organizaciones saharauis en España de carácter político, social y cultural es una prueba directa de los cambios internos que se están produciendo en la sociedad saharaui y de la importancia que han ido adquiriendo en ellos los procesos y dinámicas migratorias.

El presente artículo consta de un desarrollo en tres partes. En primer lugar, se aborda el contexto y las características principales de la migración saharaui en España, ya que la propia naturaleza del proceso de movilidad de los saharauis permite entender las razones de la formación de estas organizaciones sociales en el espacio migratorio. En segundo lugar, se describe las principales formas que toman dichas organizaciones en España: asociaciones de migrantes, colectivos de jóvenes estudiantes y colectivos culturales, analizando las relaciones - de cooperación o conflictivas - que han ido desarrollando cada una de ellas frente a las instituciones de la República Árabe Saharaui Democrática (RASD) o del Frente Polisario. ${ }^{1}$ Finalmente, se analizan los principales éxitos y debilidades de estas organizaciones, así como los problemas principales que ha tenido para constituirse y desarrollarse en España.

\section{La migración como proceso de dinamización de la sociedad saharaui}

Desde mediados de los setenta del siglo pasado la sociedad saharaui ha vivido distintas etapas; todas ellas condicionadas fuertemente por el proceso inacabado de descolonización española, el conflicto armado con Marruecos y Mauritania y el periodo de postconflicto, este último marcado por un bloqueo político sempiterno que impide una solución justa para los saharauis. Cada una de estas etapas, que tiene su punto de partida en el desplazamiento forzado que supone el éxodo hacia la región de Tinduf (Argelia) en 1975, ha generado posteriormente distintas formas de movilidad geográfica

\footnotetext{
${ }^{1}$ Frente Popular de Saguia el Hamra y Rio de Oro. Movimiento de liberación nacional saharaui constituido en 1973 con el objetivo de llevar a cabo la descolonización del Sáhara Occidental, en manos de España, y posteriormente de luchar frente a Marruecos y Mauritania, tras la ocupación del territorio en 1975.
} 
vinculadas a los estudios o a las motivaciones económicas y de mejora de las condiciones de vida. Cada una de estas formas de movilidad han ido modelando, a su vez, las características sociales, políticas, económicas y culturales que distinguen actualmente a los saharauis, extrayéndolos de la visión excesivamente estática, victimizada y dependiente que se ha ido generando sobre ellos desde los movimientos de solidaridad europeos (principalmente españoles) y desde la propia academia, tras cuarenta años de emplazamiento en los campamentos de refugiados.

Entre finales de la década de los setenta y comienzos de los ochenta, en plena guerra entre Marruecos y la guerrilla del Frente Polisario, varios miles de niños saharauis salieron de los campamentos de refugiados hacia distintos países con los que el Frente Polisario había concluido acuerdos en materia de educación ${ }^{2}$ para que formaran a las élites participantes en la construcción futura del Estado saharaui (Gómez, 2011; Corbet, 2006).

Para muchos de estos niños suponía, en muy poco tiempo, la consecución de un segundo desplazamiento, no menos traumático que el anterior, por cuanto implicaba una separación radical de sus familias durante un largo periodo de tiempo. En el caso de los niños que fueron elegidos para hacer sus estudios en Cuba, las estancias de estudio se prolongaron una media de entre seis y quince años por lo que el retorno hacia los campos de refugiados a lo largo de la década de los noventa -ya como jóvenes adultos- fue cuanto menos difícil para muchos de ellos (Corbet, 2008). El retorno, entendido como proceso de movilidad, con sus rupturas y acomodamientos con respecto a la fase anterior, genera enormes expectativas para la primera generación de jóvenes saharauis que hicieron sus estudios en el exterior, ya que se produce en paralelo a la firma de los acuerdos de paz entre el Frente Polisario y Marruecos (1991), es decir, bajo la esperanza que genera el posible desarrollo de un referéndum de autodeterminación.

Los dos procesos de movilidad anteriormente descritos: estudios y retorno a los campamentos, consolidan una experiencia de la movilidad distinta a la inscrita tradicionalmente en la sociedad saharaui y su cultura beduina. Es decir, una experiencia ligada esta vez a la lucha por la liberación nacional y el sacrificio colectivo. No es extraño, por lo tanto, que a mediados de los años noventa, cuando el bloqueo de la realización del referéndum es más que una evidencia y se suceden los fracasos de las negociaciones diplomáticas en la arena internacional (Acuerdos de Houston, 1997; Plan Baker I, 2001; Plan Baker II, $2003)^{3}$, esta generación de jóvenes altamente cualificada, sea la primera también en emprender un nuevo proceso de movilidad fuera de los campamentos, lo que ellos llaman su "tercer exilio" (Corbet

\footnotetext{
${ }^{2}$ Se trata principalmente de Cuba, Argelia, Libia, Siria y varios países de Europa del Este (la antigua Checoslovaquia y Polonia).

${ }^{3}$ Ver los trabajos de Fernández Árias (2005) y López García (2007)
} 
2006; Gómez 2011); el cual corresponde a las migraciones de carácter económico que toman como dirección principal la antigua metrópolis, España. ${ }^{4}$

Los lazos históricos con la antigua colonia hacen pensar a los migrantes saharauis que ésta podría garantizarles cierta protección y un trato preferencial en la regularización de sus situación administrativa. Sin embargo, su llegada se vio salpicada desde el inicio por nuevas decepciones: una sociedad desconfiada y xenófoba con las poblaciones procedentes del Magreb; una administración y un sistema jurídico que hacía tabula rasa de los lazos históricos entre España y el Sáhara occidental, desconociendo -como si lo hace con otras poblaciones- ciertas prioridades en la obtención más rápida de la nacionalidad española (Gómez 2011; Ruíz Miguel, 1995; 1999a, 1999b); fuertes obstáculos con respecto a la homologación de sus diplomas de estudio y una inmersión obligada en la economía irregular y en empleos muy alejados de su alta cualificación.

Más allá de los problemas relativos a un proceso migratorio, que además se produce con cuentagotas $^{5}$, la decisión de la migración se ve atravesada por profundos sentimientos de culpabilidad que supone dejar a las familias de nuevo en los campamentos, como ya pasó cuando eran niños. La presión es doble y la genera tanto la sociedad como las autoridades saharauis que, en un principio perciben la migración como una forma de traición a la causa y a los esfuerzos realizados para formarles como futuras élites del Estado saharaui. En cierta medida, su salida no solo rompe con el proyecto educativo conformado durante varias décadas por el Frente Polisario sino que introduce el miedo a un posible vaciamiento de los campamentos. Ante estas ideas, los jóvenes se ven forzados a justificar la migración a través de un triple compromiso que condiciona las características de la propia migración: el retorno a los campamentos cuando sea necesario, lo que transforma la instalación en España en algo temporal; el mantenimiento de la unidad del pueblo saharaui en el espacio diaspórico; y el trabajo por la causa en los lugares de instalación (Gómez 2010).

Aunque más adelante la llegada de remesas cambiará profundamente la concepción primigenia sobre la migración económica en el seno de los campamentos, las presiones ejercidas sobre estas primeras migraciones (1996-1997) y el contexto limitante de esta triple promesa condicionan las

\footnotetext{
${ }^{4}$ No existen cifras oficiales sobre el número de saharauis residentes en el país. Las informaciones que se recogieron durante el trabajo de campo de mi tesis doctoral situaban aproximadamente el número de saharauis en España entre 10.000 y 12.000 personas. Estas cifras han podido cambiar considerablemente a lo largo de los últimos 6 años a causa de la crisis económica y de los retornos hacia los campamentos de refugiados que ésta pudo desencadenar (Gómez, 2011).

${ }^{5}$ Con respecto a aquellos que salen de los campamentos, la migración está enteramente controlada por el Frente Polisario, que facilita o no la salida de la población, haciendo de intermediario con la administración argelina, encargada de entregar pasaportes especiales a los saharauis. La embajada española en Argel se encarga de la expedición de los visados y la red migratoria se ocupa de pagar el viaje en avión desde Tinduf (Gómez, 2011)
} 
relaciones de los migrantes con las autoridades saharauis, lo que termina reflejándose en la forma particular en el que se desarrollan las organizaciones sociales y políticas saharauis en España.

\section{La multiplicación de organizaciones saharauis en el espacio migratorio}

Como se indicaba más arriba, los primeros años de la llegada a España son difíciles. Los lazos entre los migrantes son bastante débiles, su número es aún muy reducido y, además, los recién llegados no cuentan con el apoyo ni del Frente Polisario ni de la potente red de solidaridad española con la causa saharaui. La migración no es vista con buenos ojos los primeros años y ambos actores concentran además sus esfuerzos en los campamentos de refugiados. No son pocos los discursos, en este sentido, que con un cierto malestar emplazan a los migrantes a que se responsabilicen de su propia estancia en España (Gómez, 2011).

La formación de una organización que brinde apoyo a los migrantes saharauis no aparece hasta el año 2004. En este momento la inversión en una red asociativa propia se vuelve una prioridad para los migrantes que necesitan con urgencia la constitución de una estructura intermediaria entre la administración española y saharaui capaz de velar por sus intereses.

A pesar de sus estructuras aún débiles, las organizaciones saharauis adquieren diversas formas: asociaciones de migrantes, colectivos de jóvenes universitarios y grupos culturales y de intelectuales. ${ }^{6}$ Estas organizaciones surgen con un doble objetivo. Por un lado, activar los contactos y las redes de información y apoyo entre los migrantes instalados en España para poder llenar el vacío de representación que existía en el espacio migratorio (cumplimiento de la segunda promesa). Por otra parte, completar el trabajo del Frente Polisario y del movimiento de solidaridad para hacer visible la problemática del Sáhara Occidental en España (cumplimiento de la tercera promesa).

\subsection{Las asociaciones de migrantes}

Los primeros intentos de formación de estructuras asociativas tienen lugar en las Islas Canarias, en la Comunidad de Madrid y en Cataluña. Sus primeros pasos son sin embargo tambaleantes debido a las reticencias expresadas por el Frente Polisario a que estas organizaciones se constituyan por fuera del organigrama oficial del movimiento de liberación nacional. No obstante, en 2004, cuando la migración deviene una realidad innegable, el Frente Polisario termina aceptando la constitución de las asociaciones

\footnotetext{
${ }^{6}$ En el transcurso del trabajo de campo de la tesis entrevisté a cuatro presidentes de asociaciones de migrantes saharauis en Galicia, Cataluña, País Vasco y Valencia; dos representantes de los colectivos de jóvenes: « Jóvenes por una causa libre » y " Colectivo de la juventud saharaui en España»; finalmente, también me entrevisté con cuatro miembros del grupo de intelectuales y escritores saharauis « La generación de la amistad».
} 
de migrantes saharauis en España; una decisión que se ve alentada, en parte, por los beneficios económicos que la migración estaba reportando en los campamentos de refugiados.

Por otra parte, la temida idea del vaciamiento de los campamentos no se estaba produciendo, por lo que la revalorización de los migrantes empieza a articularse en torno a diversos cambios: la aceptación de que la asociaciones gocen de una cierta autonomía de acción con respecto a la estructura oficial del Frente Polisario y sus organizaciones de masas ${ }^{7}$; una mayor coordinación entre los delegados del Frente Polisario y las asociaciones, facilitando la gestión de ciertos procesos jurídico-administrativos en España; la constitución de una oficina del Frente Polisario en Madrid dedicada por entero al tema migratorio; y, finalmente, la incorporación de representantes de los migrantes saharauis en los Congreso del Frente Polisario, concretamente desde el XII Congreso que tiene lugar en diciembre de 2007.

En el transcurso de los años varias asociaciones de migrantes van apareciendo de forma independiente por distintas regiones españolas. Las más activas hasta el año $2013^{8}$ son las del País Vasco, la Comunidad Valenciana, Cataluña, Aragón y las Islas Canarias. A pesar de sus problemas de consolidación, los primeros años de vida de estas asociaciones están marcados por el optimismo. El compromiso de trabajo por la causa se instala como el principal argumento de atracción de la población migrante hacia las asociaciones y, al mismo tiempo, dicho discurso también les confiere una legitimidad incontestable frente a las reticencias aun existentes entre las autoridades saharauis.

Estas asociaciones se transforman en puntos de referencia para los migrantes saharauis, en lugares de reunión, de discusión y de intercambio de información sobre temas variados: procesos de regularización, obtención de la nacionalidad española, acogida e información sobre alojamiento, información sobre ofertas de empleo y procesos de homologación de títulos, organización de cursos de lengua, eventos culturales y fiestas, etc. Incluso algunas de estas asociaciones se encargan también de los procesos burocráticos en torno a los fallecimientos de saharauis en el extranjero y su repatriación a los campamentos de refugiados (Gómez, 2011). En definitiva, estas organizaciones han tratado de combinar las acciones centradas en el proceso migratorio con otras de tipo cultural o más político.

En el contexto de irrupción marcado por el optimismo se asiste en la segunda mitad de la década de los 2000 a una multiplicación de las asociaciones. No obstante, la mayor parte de ellas no consiguen consolidarse verdaderamente, ni en cuanto al número de miembros ni en cuanto a la relevancia de sus actividades. Desde su creación, los problemas fueron erosionando el entusiasmo que caracterizó los primeros años. Primeramente, porque todas las asociaciones no avanzaron con el mismo impulso, muchas de ellas se encontraron aisladas, lo que produjo una dispersión de sus fuerzas o funcionaron

\footnotetext{
7 Se trata de la Unión Nacional de Mujeres Saharauis (UNMS), la Unión de Jóvenes de Saguia el Hamra et Rio de Oro (UJSARIO) y la Unión General de Trabajadores de Saguia el Hamra et Rio de Oro (UGTSARIO).

${ }^{8}$ Momento en el que realicé mi último trabajo de campo con respecto a esta cuestión.
} 
exclusivamente por el esfuerzo en solitario de algunos individuos más comprometidos con la causa. Por otra parte, la falta de lugares para celebrar los encuentros, la ausencia de financiamiento o de apoyos reales del Frente Polisario y de la red de solidaridad, e incluso la movilidad constante de los saharauis dentro y fuera de España -más aun con la crisis económica que generó el retorno de numerosos saharauis a los campamentos- ha hecho extremadamente difícil el mantenimiento o la consolidación de estas asociaciones.

\subsection{Los colectivos de jóvenes universitarios}

Entre 2006 y 2007 irrumpen en el escenario migratorio varios colectivos a iniciativa de jóvenes saharauis insertos en institutos y universidades españolas. Se trata de jóvenes residentes en España desde hace varios años, muchos de ellos pertenecientes al programa "Vacaciones en Paz"9 que pudieron quedarse en el país con posterioridad.

Entre 2006 y 2009 pueden distinguirse dos colectivos principales: Jóvenes por una causa libre (constituido aproximadamente por treinta personas) y Colectivo de la juventud saharaui (COJUVSA) (conformado por una decena de personas) que funcionan como precursores de la movilización social y política de los jóvenes saharauis en España. Ninguna de estas dos primeras experiencias se desarrolla más allá de una fase embrionaria y su capacidad de acción es más bien limitada. No obstante, su surgimiento es fundamental, pues supone un revulsivo para la conformación posterior de organizaciones de jóvenes más potentes y estructuradas. La idea principal que movilizan ambos colectivos es la unión de la juventud saharaui de la diáspora en torno a organizaciones de lucha por la causa. Como explicaba Hamidou, estudiante de marketing internacional en el País Vasco y miembro fundador del COJUVSA,

La falta de contacto con la realidad saharaui para los chicos que están en España desde hace mucho tiempo provoca que olviden de donde vienen... que han nacido en un campamento de refugiados ${ }^{10}$

En este momento es fundamentalmente el colectivo Jóvenes por una causa libre el que tiene más éxito, pues supo incorporar en su seno tres corrientes distintas de interpretación de la lucha de los

\footnotetext{
${ }^{9}$ Se trata de una iniciativa de las asociaciones pro-saharauis en España en coordinación con el Frente Polisario y diversas instituciones públicas españolas (ayuntamientos, subdelegaciones provinciales del gobierno, etc.,) destinada a niños saharauis entre cinco y doce años. El programa comienza en los años ochenta pero crece considerablemente a partir de la década de los noventa. Surge con el objetivo de alejar a los niños saharauis de los campamentos durante los meses de verano y poder hacerles chequeos médicos completos. Aunque el programa no está destinado a niños mayores de doce años, muchos de aquellos que se beneficiaron del mismo acabaron insertos en otros programas de acogida bajo acuerdos entre las familias en los campamentos y las familias de acogida en España. En algunos casos estos procesos han dado lugar con el tiempo a diversos problemas y tensiones relacionados con la adopción de estos niños.

${ }^{10}$ Entrevista realizada en Bilbao en enero de 2009.
} 
jóvenes en el contexto migratorio. ${ }^{11}$ Su funcionamiento en red y el apoyo que encuentra en las nuevas tecnologías de la comunicación y la información le permiten tejer mejor los contactos con los jóvenes que desean participar en las actividades reivindicativas y de información sobre el conflicto saharaui, especialmente en el ámbito estudiantil de institutos y universidades.

Contrariamente a la evolución de las asociaciones de migrantes, los colectivos de jóvenes han conocido un verdadero auge desde 2010. Aunque la actividad de los dos colectivos primeros se pierde en los últimos años, su trabajo conduce a la irrupción de nuevas iniciativas dirigidas por estudiantes, principalmente universitarios, mucho más coordinadas y conectadas las unas con las otras, evitando así la dispersión de fuerzas o el aislamiento.

Durante el XII Congreso del Frente Polisario organizado en diciembre 2007, la juventud saharaui toma consciencia de la existencia de una diversidad de grupos dentro de los campamentos de refugiados, en los territorios ocupados y en España que se han desarrollado al calor de la llamada intifada saharaui de 2005, y que tienen un perfil similar y luchas políticas convergentes. Los colectivos que surgen en España en esta época, reivindican la conexión con esas otras organizaciones que permita la unión de los jóvenes estudiantes saharauis. No es sin embargo hasta el año 2011, en el transcurso del XIII Congreso del Frente Polisario que se materializan las aspiraciones de unión a través del surgimiento de una nueva organización de masas: la Unión de Estudiantes de Saguia el-Hamra y Rio de Oro (UESARIO), que supone una ruptura radical con la antigua organización de jóvenes del Frente Polisario, la UJSARIO, objeto de críticas constantes como consecuencia de su supuesta falta de iniciativa e incapacidad para adaptarse a las exigencias y necesidades de los jóvenes migrantes.

La UESARIO nace con el objetivo de unificar a los jóvenes y los movimientos de estudiantes en los tres espacios principales de instalación de los saharauis: campamentos, territorios ocupados y España. En este último lugar la organización se encuentra representada por la Liga de estudiantes y jóvenes saharauis en el Estado Español (LEJSEE), constituida en 2012. Existen igualmente varias asociaciones de jóvenes dispersas en diversas regiones españolas, las dos más activas son las de Cataluña y las Islas Canarias. Por otra parte, el colectivo universitario Legrif se emplaza como punto de referencia después de su constitución en 2014. El macro encuentro de jóvenes universitarios saharauis organizado por este colectivo en febrero de 2016 en Zaragoza es una prueba de ello. El encuentro visibiliza la amplitud e importancia que empiezan a adquirir los jóvenes saharauis residentes en España y sus contribuciones a la

\footnotetext{
${ }^{11}$ La primera, minoritaria, entiende el colectivo como un medio de aunar a los jóvenes saharauis en torno a la cultura tradicional y de recuperar a los jóvenes desarraigados. La segunda corriente, igualmente minoritaria, pero mucho más radical tanto en su discurso como en las acciones que trata de realizar (autonomía completa con respecto al frente Polisario, imperativo de vuelta a las armas, etc.), entiende el colectivo como una plataforma de contestación y de crítica sin concesiones con respecto al gobierno saharaui. Finalmente, una tercera corriente, mayoritaria, se emplaza como pieza fundamental de unión entre las otras dos valorizando principalmente el trabajo colectivo frente al olvido.
} 
causa nacional. Es una muestra igualmente del dinamismo social que caracteriza hoy por hoy a la sociedad saharaui, de la diversidad de los emplazamientos en donde se produce la discusión, del sentido que adquieren unas reivindicaciones alejadas espacialmente de los dos principales territorios productores de sentido (campamentos de refugiados y territorios ocupados), del surgimiento de una nueva generación con otras perspectivas, otras formas de pensar el conflicto arraigadas en su propia experiencia vital, diversa a la de sus padres y abuelos.

\subsection{El grupo de intelectuales y escritores "La Generación de la Amistad"}

La última de las organizaciones a señalar ha conocido desde sus inicios una evolución positiva y bastante exitosa. Se trata de La Generación de la amistad ${ }^{2}$, un grupo de intelectuales y escritores saharauis constituido por un núcleo de once personas y diversos colaboradores, la mayoría pertenecientes a la primera generación de niños saharauis que realizaron sus estudios en Cuba.

De todas las organizaciones surgidas en el contexto migratorio la que ha conseguido con mayor éxito visibilizar la historia y la cultura saharaui a través de la literatura. Gracias a su recorrido, el Frente Polisario ha podido exportar una imagen ejemplar de la población saharaui, ya que se trata de un grupo con una formación académica alta, un conocimiento perfecto de la lengua y la cultura española y un compromiso firme con la causa. La escritura supone para estos saharauis un "archivo de memoria", el medio más eficaz de transmisión y de defensa de una cultura fundamentalmente de carácter oral que se encuentra en seria amenaza de desaparición en la actualidad. En este sentido, la literatura se entiende como un instrumento de lucha frente al olvido y la invisibilidad impuestos por el Estado español y sus principales instituciones culturales (Gómez, 2013).

Más allá de los congresos y jornadas de estudio organizadas por diversas universidades españolas y sus obras colectivas e individuales, su principal medio de difusión y acceso a un público más amplio ha sido internet. El grupo gestiona una página web y tres blogs: La Generación de la amistad, Poemario por un Sahara libre y Tiris novia de poetas. Estas páginas tienen importantes contenidos sobre eventos culturales y académicos relacionados con el Sáhara Occidental y noticias sobre los campamentos de refugiados y los territorios ocupados.

\footnotetext{
${ }^{12}$ La Generación de la Amistad hace parte de la denominada “segunda generación Polisario”. Estos jóvenes tomarán el relevo de un primer grupo de escritores y poetas saharauis percusores de la literatura saharaui en español. Este primer grupo, llamado también la generación de vanguardia, estudió en las universidades españolas a finales de la década de los sesenta y principios de los setenta. La mayoría de ellos se enroló en el Frente Polisario en 1975 y murió durante la guerra (Awah 2008).
} 


\section{4. Éxitos y desafíos de las organizaciones sociales, políticas y culturales saharauis en España}

Aunque la evolución de todas las organizaciones ha sido bastante rápida, su desarrollo posterior presenta importantes diferencias. Mientras que las primeras habían vivido un crecimiento considerable desde 2004, seguidas a partir de 2010 de una cierta retracción (como consecuencia del aislamiento de unas asociaciones con respecto a otras, la falta de apoyos, la crisis económica en España, etc.,), los colectivos de jóvenes y el grupo de intelectuales supieron tejer lazos mucho más sólidos. Esto ha permitido tanto su diversificación como su consolidación y consideración como puntos de referencia de la lucha saharaui por fuera de los campamentos de refugiados. La ruptura del aislamiento que protagonizan los colectivos de jóvenes y el grupo de escritores (dialogando con otros colectivos o escritores no solamente saharauis sino también españoles) ha sido fundamental para que estas organizaciones puedan desplegarse, construir redes más firmes y conseguir visibilidad pública.

Con respecto a las asociaciones de migrantes, su principal aspiración era actuar a nivel social y cultural en el seno de la comunidad de migrantes y, en la medida de lo posible, dentro de la sociedad española. En este sentido, consiguieron en un primer momento ayudar a los nuevos llegados, ofrecerles cierta protección y, sobretodo, mostrar que, a pesar de los numerosos problemas y las faltas de apoyo, la auto-organización era posible. El objetivo de su constitución nunca fue político, sin embargo nunca pudieron alejarse del campo político. El hecho de querer mantener una autonomía de acción y de trabajar por fuera de las estructuras oficiales del Frente Polisario, les transformó en referencia política para los colectivos de jóvenes que se conforman posteriormente.

Como indicaba, Ibrahim, trabajador en la intermediación cultural en un centro de jóvenes migrantes en Cataluña. La oposición de la asociación de migrantes saharauis en esta región con respecto a una posible absorción de la organización por la dirección del Frente Polisario era palpable:

Nosotros acogimos con alegría la concesión que hizo el Polisario de ciertas de las reivindicaciones que hacíamos. Pero eso...eso no quiere decir que con los nuevos gestos acabara poniendo a las asociaciones bajo su control. La experiencia de nosotros debe de ser entendida como algo positivo, incluso por el gobierno saharaui. Me gustaría señalar que el movimiento asociativo nos ha permitido actuar dentro de un ámbito de transparencia democrática, tanto en las elecciones de nuestros representantes como en las decisiones que tomamos. Digamos que estamos experimentando en nosotros mismos el sistema de poder que quisiéramos para un futuro estado saharaui. ${ }^{13}$

Cabe preguntarse, sin embargo, por qué el Frente Polisario acabó aceptando que una parte de los jóvenes saharauis, muchos de ellos altamente cualificados, se instalara en España y se organizara independientemente de sus estructuras oficiales. La respuesta probablemente está ligada al contexto

\footnotetext{
${ }^{13}$ Entrevista realizada el 22 de noviembre de 2007 en Barcelona.
} 
social de los campamentos de refugiados. La migración ha permitido alejar a los individuos potencialmente contestatarios de los campamentos, mientras que sus aportes económicos han supuesto también un elemento de contención social al abrir nuevas posibilidades de existencia más allá de las donaciones procedentes de las organizaciones internacionales y de las asociaciones de solidaridad. De esta forma, la aceptación de la migración por parte del gobierno saharaui supuso en realidad una bocanada de oxígeno a una población con evidentes carencias y muy frustrada por los fracasos sucesivos de la diplomacia saharaui y la comunidad internacional (Gómez y Omet, 2009).

Los aportes económicos realizados por los migrantes les dan un cierto poder de negociación, reforzando su posición con respecto a la autonomía de acción en el espacio migratorio y el plegamiento del Polisario a ciertas de sus reivindicaciones. Este posicionamiento del Polisario no debe ser interpretado, sin embargo, como un signo de debilidad. Por el contrario, el realizar estas concesiones le ha dado más tiempo para habituarse a la nueva situación y para reforzar su dominación en un campo político que se encuentra en clara mutación, con una diversidad de nuevos actores que quieren participar.

Con respecto a las posibilidades de transformación de la hegemonía en el espacio político saharaui, los colectivos de jóvenes han sabido analizar la situación más claramente que las asociaciones de migrantes, que se han conformado simplemente con tener representantes en los congresos del Frente Polisario. Después de su constitución, los colectivos de jóvenes, han vivido un doble proceso de politización tanto en el campo migratorio como en la arena política saharaui. Por un lado, los jóvenes no han dudado en señalar que la migración no ha estado a la altura del compromiso con la causa (es decir, con la tercera promesa de la migración referida anteriormente) y que, en ese sentido, ha habido una clara falta de iniciativa de los migrantes y de los jóvenes estudiantes en España. Más allá de acciones puntuales: académicas, socio-culturales o políticas, reivindican principalmente el retorno al espíritu combativo que había caracterizado a la población saharaui durante los años de guerra, por lo que exigen un apoyo más firme a las luchas de los jóvenes saharauis en los territorios ocupados. Su firme compromiso con la causa les ha permitido legitimar su presencia en una sociedad en plena evolución y en la cual este discurso de resistencia es ampliamente aceptado.

Por otro lado, los jóvenes han apostado por conquistar, en nombre de la libertad de expresión, ciertos espacios de acción en el seno del Frente Polisario (lo que significa que nunca han cuestionado la legitimidad de esta organización y que siguen considerándolo como el principal instrumento de lucha). De hecho, sus aspiraciones -tanto dentro como fuera de los campamentos- es poder reemplazar progresivamente a los antiguos cuadros políticos y las estructuras rígidas del gobierno, adoptando responsabilidades progresivamente. De esta manera han elaborado una estrategia de adelantamiento de 
la organización clásica de jóvenes del Frente Polisario, UJSARIO ${ }^{14}$. Como se indicó anteriormente, la irrupción en 2011 de la UESARIO es una prueba de la inversión de esfuerzos en el campo político y de la seriedad de su proyecto.

A pesar de la evolución positiva que han tenido varias de estas organizaciones, lo cierto es que, en términos de movilización social y política, la acción de los saharauis de la diáspora es todavía bastante débil y limitada. Dos factores han impedido particularmente un desarrollo más fuerte de las mismas. En primer lugar, la inexperiencia por fuera de los límites fijados por el Frente Polisario. A partir de los años noventa, el retorno a los campamentos de los hombres que habían participado en la guerra y la situación frustrante de "ni paz ni guerra" deja a la población saharaui en una situación de apatía y de espera. Con el tiempo se produce además una transferencia generalizada de responsabilidad con respecto a la organización y la estructuración de los campamentos -antes en manos de las mujeres saharauis- a los cuadros de la administración de la RADS y a la red asociativa pro-saharaui, encargada de la ayuda humanitaria y el desarrollo de infraestructuras en los campamentos.

En segundo lugar, los migrantes se han enfrentado constantemente con el miedo de ser acusados de traición. Durante los primeros años de la década del 2000 asistimos a la irrupción tanto en los territorios ocupados como en España de varios movimientos, organizaciones e incluso partidos políticos como El Consejo Real Consultivo para los asuntos del Sáhara (CORCAS) o el Grupo Saharaui Independiente (Islas Canarias) constituidos por marroquís o por saharauis pro-marroquís. Su constitución hace parte de una política clara de la monarquía alauita que busca desestabilizar al Frente Polisario intentando introducir la duda sobre su legitimidad como único representante del pueblo saharaui. Esto hecho generó una especie de psicosis en el seno del movimiento de liberación nacional saharaui y las organizaciones españolas de solidaridad, lo que explica por qué cada vez que las asociaciones de migrantes y los colectivos de jóvenes intentan desarrollar actividades en nombre de una supuesta comunidad saharaui en España, deben repetir incesantemente que sus objetivos no están en hacerle la competencia al Frente Polisario o de poner en cuestión su legitimidad. La sola sospecha limita, por lo tanto, su capacidad de acción.

Para estas organizaciones, sin embargo, el objetivo es incorporar nuevas voces y nuevos actores en el seno del Frente Polisario, saltar de los márgenes hacia el centro y hacer del Frente Polisario una organización más democrática y adaptada al dinamismo de su sociedad. Una sociedad que ha evolucionado presionada por el ritmo apático de la espera en los campamentos y, al mismo tiempo, por la necesidad de sobrevivir, de reinventarse, de proyectarse en un futuro diferente que el exilio en los campamentos de refugiados.

\footnotetext{
14 Para mayor información sobre la estructuración de una oposición todavía tímida de una parte de la juventud de los campamentos de refugiados y de la diáspora ver Gómez y Omet (2009).
} 


\section{Conclusiones}

La acumulación de estas prácticas, experiencias y discursos sociales, políticos y culturales diferentes ha dado nacimiento en los últimos diez años a nuevas formas de organización, especialmente en el espacio migratorio. A pesar de la dificultad de su desarrollo y las tensiones que se han ido materializando después de su irrupción en el campo político saharaui, estas organizaciones reflejan una nueva configuración social que comienza a exigir la adaptación de las tradicionales estructuras del Frente Polisario a la realidad actual, muy alejada ya de los esquemas de acción y de lucha de décadas precedentes.

El mayor éxito de estas organizaciones ha sido precisamente visibilizar los cambios y la evolución constante tanto de la sociedad saharaui como de su campo político. Ellas son también, sin ninguna duda, el símbolo más claro de concienciación de los migrantes, incluyendo los jóvenes estudiantes, del rol principal como actores económicos y políticos que están destinados a cumplir tanto en clave presente como futura. El Frente Polisario sigue siendo sin objeciones el único instrumento legítimo de lucha en la consecución de un Estado saharaui pero también se vuelve el centro de variadas peticiones -igualmente legítimas- que implican su apertura a nuevas formas de participación y de lucha en estos primeros impasses del siglo XXI. 


\section{Bibliografía}

Awah, B. (2008). Literatura del Sáhara Occidental: un esbozo histórico. Madrid: BABOK.

Corbet A. "Les campements de réfugiés sahraouis en Algérie : de l’idéel au réel”. Bulletin de l'Association de Géographes Français 83/1 (2006): 9-21.

Corbet A. (2008). Nés dans les camps. Changements identitaires de la nouvelle génération de réfugiés sahraouis et transformations des camps. Tesis de Antropología, París, EHESS.

Gómez Martín, C; Omet, C. "Les cheminements de la politisation des jeunes dans le champ politique Sahraoui. Etude comparative entre la diaspora et les camps de réfugiés “. L'Année du Maghreb, V (2009): 205-222.

Gómez Martín C. (2010). "Saharauis: una migración circular entre España y los campamentos de refugiados de Tinduf”. de Castro, C. (ed.), Mediterráneo Migrante. Tres décadas de flujos migratorios. Murcia: Universidad de Murcia.

Gómez Martín C. (2011). La migración saharaui en España. Estrategias de visibilidad en el tercer tiempo del exilio, Editorial Académica Española, Saarbrücken, Alemania.

Gómez Martín C. (2013). "La literatura saharaui contemporánea y su desarrollo en el espacio migratorio español”. RIPS 12/2 (2013): 229-241.

Gómez Martín C. (2016). “De exilios y migraciones económicas. La movilidad espacial de los saharauis como parte del "ingenio aguzado de la supervivencia”. Barreñada, I. y Ojeda, R (eds.), Sáhara Occidental. 40 años después. Madrid: Ediciones la Catarata.

López García, B. “Iniciativas de negociación en el Sáhara Occidental: historia de la búsqueda de una "solución política"”. ARI85 (2007).

Fernández Arias, C. "El Sáhara Occidental: un año después de Baker”. Política Exterior 107, (2005): 74-76.

Ruíz Miguel, G.(1995). El Sáhara Occidental y España: Historia, Política y Derecho. Análisis crítico de la política exterior española. Madrid: Dykinson.

Ruíz Miguel, C. "Nacionalidad española de los ciudadanos saharauis: secuela de una descolonización frustrada (y frustrante)". Revista General de Derecho 663 (1999a):14235-14245.

Ruíz Miguel, C.. "Nacionalidad, igualdad y descolonización. Comentario a la STS (Sala 1a) de 28 de octubre de 1998”. Revista Española de Derecho Constitucional 56 (1999b): 251-278. 